\title{
Progress in the Treatment of Hematological Diseases with Rituximab
}

\author{
Xiangjun Fu, Li'e Lin, Hongxia Yao, Li Huang, Can Meng, \\ Shuwen Wang, Dan Liu, Li Guo*, Mengling Duan \\ Hainan General Hospital, Haikou, China \\ Email: ^g2002m@163.com
}

How to cite this paper: Fu, X.J., Lin, L.E., Yao, H.X., Huang, L., Meng, C., Wang, S.W., Liu, D., Guo, L. and Duan, M.L. (2021) Progress in the Treatment of Hematological Diseases with Rituximab. Open Journal of Blood Diseases, 11, 6-14.

https://doi.org/10.4236/ojbd.2021.111002

Received: January 17, 2021

Accepted: February 26, 2021

Published: March 1, 2021

Copyright $\odot 2021$ by author(s) and Scientific Research Publishing Inc. This work is licensed under the Creative Commons Attribution International License (CC BY 4.0). http://creativecommons.org/licenses/by/4.0/

\begin{abstract}
Rituximab is a mouse and human chimeric $\mathrm{CD}_{20}$ (anti-B cell) specific monoclonal antibody that has been approved by the U.S. Food and Drug Administration for the treatment of lymphoma. The expression of $\mathrm{CD}_{20}$ antigen is expressed in the whole ontogeny of $B$ cells, starting from the premature $B$ cells in bone marrow to the differentiation of plasma cells in secondary lymphoid tissues. The wide distribution of $\mathrm{CD}_{20}$ molecules allows rituximab to eliminate a large number of $\mathrm{B}$ cells. Rituximab is the core drug for the treatment of hematological diseases, often combined with drugs as a first-line treatment. Long-term hormone therapy often results in serious adverse reactions, and new therapies, which can avoid widespread immunotoxicity, have great potential for treating diseases of the blood system.
\end{abstract}

\section{Keywords}

Rituximab, Malignant Lymphoma, Leukemia, Multiple Myeloma, Autoimmune Diseases, Hematopoietic Stem Cell Transplantation

\section{Introduction}

Rituximab, a genetically engineered chimeric mouse/human IgG1- $\kappa$-anti-CD ${ }_{20}$ monoclonal antibody (mAb), has been approved by the U.S. Food and Drug Administration for the treatment of various subtypes of B-cell non-Hodgkin's lymphoma (NHL). Clinical studies have demonstrated the efficacy of rituximab in the treatment of autoimmune diseases, such as rheumatoid arthritis [1] [2] and immune thrombocytopenic purpura (ITP), etc. [3]. Morath C et al. treated refractory thrombocytopenia and allogeneic SCT with rituximab in oral and ocular manifestations of cGVHD, and found that in addition to improved platelet count, cGVHD symptoms were also reduced and immunosuppression was 
successfully stopped [4]. Subsequent small case series have shown mixed results of steroid-resistant cGVHD [5]-[11]. Thus, the induction of new mechanisms of cGVHD, including high activation and aberration of donor B cells, paves the way for new therapeutic modalities that ultimately improve allogeneic HSCT outcomes. In recent years, with the deepening understanding of B lymphocytes and their mechanism of action, the therapeutic scope of rituximab has expanded from B cell malignant lymphoma to chronic lymphocytic leukemia, multiple myeloma and a variety of autoimmune system diseases, and even plays an important role in hematopoietic stem cell transplantation. Rituximab rapidly clears $B$ cells through cytotoxic action, which interferes or even inhibits the generation of autoimmune antibodies. It is effective in treating refractory AIHA in children [12]. Rituximab was well tolerated with fewer side effects. The main side effects include: 1) acute infusion reaction: hypotension, fever, chills, chills, bronchospasm, etc. which generally appear in the first application, can gradually reduce later. 2) The incidence of serological reaction is higher in infants [13]. 3) Long-term reduction in autoantibody production can increase the risk of viral and bacterial infections. Chickenpox virus [14], enterovirus [15], and pneumocystis carinii have been found. Therefore, serum immunoglobulin levels should be monitored and prophylactic gamma globulin infusion should be given during treatment, and treatment should be given to prevent pneumocystis carinii. The progress of its clinical application is as follows.

\section{Rituximab in the Treatment of Chronic Lymphocytic Leukemia (CLL)}

Chronic lymphoblastic leukemia (CLL) is the most common leukemia in Europe and the United States [16]. The incidence in China is significantly lower than that in western countries, but it is not rare. CLL can be identified from most other lymphatic proliferation and sputum diseases of the Bin area mainly according to typical lymphocyte morphology and immunophenotype characteristics, and some patients need to be identified by combining pathology, immunohistochemistry and cytogenetics. CLL weakly expressed CD20 was detected by flow cytometry, and standard dose rituximab was used to treat recurrent CLL. The total effective rate is 10 to 15 percent, and since. The duration of effect is shorter. Recent studies have shown that the expression level of CD20 does not affect the effective rate of treatment [17]. However, the increase of free CD20 antigen in the blood circulation of CLL patients makes rituximab neutralized before reaching target cells, so the serum rituximab concentration decreases, which is related to the short effective duration of rituximab [18]. In order to improve the efficacy of rituximab, a recent clinical trial increased the dose to $2250 \mathrm{mg} / \mathrm{m}^{2}$ once a week for 4 times, with an overall response rate of up to $75 \%$. The treaty-related toxicity was similar to the standard weekly use of rituximab, and the median effective remission time was 9.5 months. Another way to improve the efficacy of rituximab is through combination chemotherapy. Michael et al. [19] 
reported a clinical study on the treatment of 224 newly treated advanced CLL patients with rituximab combined with fludarabine and cyclophosphamide (FC), and the results showed that the total effective rate was $95 \%$. Some patients who had relapsed or failed after previous fludarabine or alkylating agent treatment were still effective after receiving rituximab combined with FC regimen again [20].

Rituximab is a chimeric antibody that binds to $\mathrm{CD}_{20}$ antigen on the surface of $\mathrm{B}$ cells. Although it is highly effective for follicular lymphoma with an OR rate of $60 \%$, the single drug rituximab has poor clinical activity against B-cell CLL, which is less effective in clearing the disease from bone marrow and has a short reaction period [21] [22]. The response rate of rituximab monotherapy in previously treated CLL patients was $23 \%-45 \%$ (CR 0\% - 3\%) [21] [22]. Phase 2 studies demonstrated a dose-response correlation, and the response rate increased with the increase of rituximab dose [21]. The response rate at the dose of $500-825 \mathrm{mg} / \mathrm{m}^{2}$ was $22 \%$ (all PR), and the response rates at the dose of 1000 $1500 \mathrm{mg} / \mathrm{m}^{2}$ and the highest dose of $2250 \mathrm{mg} / \mathrm{m}^{2}$ were increased to $43 \%$ and $75 \%$ $(P=0.007)[21]$. In another study [22], rituximab was used as first-line treatment for CLL at $375 \mathrm{mg} /\left(\mathrm{m}^{2} \cdot \mathrm{W}\right)$ for 4 weeks, repeated at 6 months. The response rate after the first course of rituximab was $5 \mathrm{~L} \%(\mathrm{CR} 4 \%)$ in 44 patients with CLL or small lymphocytic lymphoma. After completion of $\geq 1$ additional course of treatment (28 cases), OR rate was 58\% (CR9\%). However, PFS at 18.6 months was inferior to that of patients with follicular lymphoma treated with first-line + maintenance rituximab [22]. The antitumor activity was increased when rituximab was combined with GM-CSF. The response rate was 79\% (CR36\%) in patients with indolent lymphoma, but $61 \%$ (CR 7\%) in patients with CLL aged $>70$ years.

\section{Rituximab in the Treatment of Autoimmune Diseases}

Blymphocytes are one of the main members of the acquired immune response. Its development process mainly goes through the stages of pre-B lymphocytes, immature B lymphocytes, initial B lymphocytes, mature B lymphocytes and memory B lymphocytes [23], and finally differentiates into bovine antibody producing plasma cells. $\mathrm{CD}_{20}^{+}$B lymphocytes participate in immunity, including: production of immunoglobulin, through which the role of immunoglobulin; B lymphocyte autoantigen presentation, regulation of other immune cells; B lymphocytes affect other antigen-presenting cells such as dendritic cells, and $\mathrm{B}$ lymphocytes can secrete cytokines such as interleukin (IL-4, IL-10) to act on other immune cells. The abnormal interaction between $\mathrm{B}$ cells and $\mathrm{T}$ cells is crucial to the occurrence and development of immune diseases. Rituximab is a monoclonal antibody targeting CD20 molecules on the surface of B lymphocytes. It can bind to CD20 molecules on the surface of cells with a high affinity, resulting in the clearance of the bound B lymphocytes and a significant reduction in the number of B lymphocytes in the body. Currently, rituximab is mainly 
used for the treatment of idiopathic thrombocytopenic purpura (ITP). Shanafelt et al. [24] retrospectively analyzed the therapeutic effect of rituximab in 12 refractory ITP patients (including 4 Evans syndrome patients), all of whom had received more than one course of conventional treatment before treatment and failed, and 6 of whom also received corticosteroids or other immunosuppressive agents during rituximab treatment. Results CR was found in 5 cases, and the therapeutic response was observed in both patients with and without splenectomy. Rituximab is a new biologic agent with relatively low therapeutic toxicity, which can play a certain role in the treatment of refractory ITP with severe bleeding symptoms, and can be used as an effective alternative therapy for patients who have failed to respond to conventional drugs. At the same time, its efficacy in ITP also reattracted more attention to the role of humoral immune mechanism in 1TP. However, the high price of rituximab limits its wide application in clinical practice. On the other hand, its specific mechanism of action and reasonable treatment plan need further study [25] [26]. Other reports rituxan in the treatment of thrombotic thrombocytopenic purpura [27], acquired VIII factor antibody increased [28] blood coagulation disorders, caused by hemolytic anemia and refractory Greave's disease has a good response to treatment.

\section{Application of Rituximab in Hematopoietic Stem Cell Transplantation}

Currently, rituximab is mainly used for in vivo purification of autologous peripheral blood hematopoietic stem cell transplantation (APBSCT). R Z et al. [29] rituxan is studied in combination with large dose of chemotherapy in patients with NHL APBSCT treatment effect, the research object and IV follicular III B non-hodgkin's lymphoma and cell lymphoma patients. The author USES the rituxan, high-dose cytarabine and mitoxantrone and g-csf in peripheral blood stem cell mobilization, separation of $\mathrm{CD}_{34}^{+}$cells, by PCR detection, after the separation cell tumor iconic harmony genes were negative, back to lose these cells, compared with control group, the hematopoietic reconstruction needed no difference between the amount of $\mathrm{CD}_{34}^{+}$cells infusion, and to rebuild hematopoietic stem cells ability between the two groups, clinical adverse reactions and no obvious difference. X L et al. [30] also confirmed that combined chemotherapy with rituximab could significantly reduce the contamination of lymphoma cells in mobilized stem cells, and the clinical and molecular remission rates of patients in the rituximab group were also significantly higher than those in the control group. Galimberti et al. [31] 23 cases of follicular non-hodgkin's lymphoma patients APBSCT, including 13 cases with CHOP plan after chemotherapy in patients with high-dose chemotherapy alone, ll patients using high doses of chemotherapy in combination with rituxan treatment, the results showed that application of rituxan treatment group of $\mathrm{Bel}-2 / \mathrm{IgH}$ rearrangement negative rate was $86 \%$, compared with high-dose chemotherapy control graft Bel-2/IgH rearrangement negative rate is $14.3 \%$, the former EFS 5 years was $100 \%$, and the lat- 
ter is only $41 \%$, This suggests that rituximab is an effective treatment for patients with follicular non-Hodgkin's lymphoma. Kosmas et al. [32] analyzed the results of several study groups and concluded that the application of rituximab did not affect the mobilization effect of 1000 cells and did not damage the function of stem cells. In vivo treatment was more effective in removing residual tumor cells than in vitro purification of monoclonal antibody. Patients with APBSCT treated with Rituximab can quickly complete graft survival. Although neutropenia occurs in individual patients, this change is reversible and may be related to the immune response. Of course, the long-term efficacy and adverse reactions of rituximab APBSCT purification therapy in vivo still need further follow-up observation.

Rituximab in the treatment of malignant lymphoma malignant lymphoma is a type of malignant tumor originating in the lymphatic hematopoietic system, and is one of the top ten most common tumors in China. It can be divided into Hodgkin's lymphoma (HL) and non-Hodgkin's lymphoma (NHL). Malignant lymphoma is a group of highly heterogeneous diseases with different clinical outcomes. Its pathological classification is complex, and the treatment principles are different for different types and different populations. Therefore, accurate assessment of the disease condition and individualized treatment should be emphasized. Chemotherapy is one of the effective methods for the treatment of malignant lymphoma, but the conventional regimen is not effective for malignant lymphoma with adverse prognostic factors. Rehwald et al. [33] a multicenter II period clinical study application of rituxan treatment of 166 cases of recurrent, refractory follicular non-hodgkin's Lymphoma non-Hodgkin's Lymphoma (NHL), by polymerase chain reaction detection of the $\mathrm{Bcl}-2$ gene rearrangement. The overall response (OR) rate was $48 \%$, the complete response (CR) rate was $6 \%$, and the median time to tumor progression was 12 months. Patients with sensitive relapsing disease still had a $40 \%$ remission rate after retreatment with rituximab, and the median time to tumor progression was 17 months. A II period, according to the clinical studies have shown that rituxan for recurrent or other subtypes of CD20 positive lymphocytes as the main type of Hodgkin's lymphoma, Hodgkin's lymphoma, NL) patients is effective. Subsequent studies have found rituximab combined with chemotherapy as a first-line regimen for the treatment of NHI. Better than chemotherapy alone. Hoehster et al. [34] 322 patients with indolent NHL CVP treatment group and CVP were followed for rituxan maintenance treatment group after treatment of progression-free survival rates were $43 \%$ and $73 \%$ respectively, total deposit rate is $89 \%$ and $96 \%$, respectively, differences were statistically significant, and two groups there was no statistically significant difference III or IV toxicity.

\section{Rituximab for Multiple Myeloma (MM)}

$\mathrm{CD}_{20}$ expression in plasma cells of $\mathrm{MM}$ patients is low in number and density, so it is necessary to increase $\mathrm{CD}_{20}$ expression on the surface of MM tumor cells in 
order to effectively attack multiple myeloma cells with rituximab [35]. The experimental results of Li Juan et al. show that when the concentration is greater than $100 \mathrm{ug} / \mathrm{ml}$ [36]. Thalidomide combined with $16 \mathrm{ug} / \mathrm{mI}$. The inhibition effect of thalidomide and rituximab alone on tumor cell colony formation in newly treated or relapsed refractory MM patients was higher than that of thalidomide and rituximab alone, indicating that thalidomide can increase the inhibition effect of rituximab on the growth of MM cells, which may be related to the up-regulation of CD20 expression in tumor cells by the former. From the above experiments, the clinical use of thalidomide and rituximab in the treatment of newly treated or recurrent refractory MM patients may be another direction of MM treatment.

\section{Conclusion}

In summary, the variable region of rituximab binds to $\mathrm{CD}_{20}$ through three different mechanisms, such as antibody-dependent cytotoxicity, complement-dependent cell killing, and induction of apoptotic cell death, which cause cell destruction and lead to persistent clearance of circulating B cells [37]. Rituximab can induce a slight decrease in anti-HLA antibodies in patients with low titer antibodies [38], which is closely related to JC virus-induced progressive multifocal leukoencephalopathy [39]. Gerberg et al. observed the pharmacodynamics of renal transplant patients after giving a single dose of rituximab, and found that it quickly eliminated B cells and persistently inhibited B cells [40]. There are also many studies using rituximab to desensitize and treat acute antibody mediated rejection (AMR) [41] [42] [43] [44].

\section{Funding}

The work was supported by Natural Science Foundation of Hainan Province (820MS137).

\section{Conflicts of Interest}

The authors declare no conflicts of interest regarding the publication of this paper.

\section{References}

[1] Kaposztas, Z., Podder, H., Mauiyyedi, S., et al. (2009) Impact of Rituximab Therapy for Treatment of Acute Humoral Rejection. Clinical Transplantation, 23, 63-73. https://doi.org/10.1111/j.1399-0012.2008.00902.x

[2] Kolb, H.-J. (2017) Hematopoietic Stem Cell Transplantation and Cellular Therapy. National Library of Medicine, 89, 267-277.

[3] Pescovitz, M.D. (2006) Rituximab, an Anti-cd20 Monoclonal Antibody: History and Mechanism of Action. American Journal of Transplantation, 6, 859-866. https://doi.org/10.1111/j.1600-6143.2006.01288.x

[4] Morath, C., Beimler, J., Opelz, G., et al. (2012) Living Donor Kidney Transplantation in Crossmatch-Positive Patients Enabled by Peritransplant Immunoadsorption 
and Anti-CD20 Therapy. Transplant International, 25, 506-513.

https://doi.org/10.1111/j.1432-2277.2012.01447.x

[5] Xia, R., Qiu, H.Y., Zhang, W.P., et al. (2005) Dynamic Monitoring of Graft-versus-Host Disease after Allogeneic Peripheral Blood Stem Cell Transplantation by Soluble HLA-I Antigen Detection. Academic Journal of the First Medical College of PLA, 25, 687-690.

[6] Okamoto, M., et al. (2006) Rituximab Is Effective for Steroid-Refractory Sclerodermatous Chronic Graft-versus-Host Disease. Transplantation Proceedings, 20, 172-173. https://doi.org/10.1038/sj.leu.2403996

[7] Carella, A.M., et al. (2007) Rituximab Is Effective for Extensive Steroid-Refractory Chronic Graft-versus-Host Disease. QJM, 48, 623-624. https://doi.org/10.1080/10428190601094362

[8] Zaja, F., et al. (2007) Treatment of Refractory Chronic GVHD with Rituximab: A GITMO Study. Haematologica, 40, 273-277. https://doi.org/10.1038/sj.bmt.1705725

[9] Mohty, M., et al. (2008) Rituximab as Salvage Therapy for Refractory Chronic GVHD. Clinical Transplants, 41, 909-911. https://doi.org/10.1038/bmt.2008.12

[10] von Bonin, M., et al. (2008) Treatment of Chronic Steroid-Refractory Graft-versus-Host Disease with Low-Dose Rituximab. Transplantation Proceedings, 86, 875-879 https://doi.org/10.1097/TP.0b013e318183f662

[11] Clavert, A., et al. (2013) Safety and Efficacy of Rituximab in Steroid-Refractory Chronic GVHD. Human Immunology, 48, 734-736.

https://doi.org/10.1038/bmt.2012.203

[12] Stasi, R. (2010) Rituximab in Autoimmune Hematologic Diseases: Not Just a Matter of B Cells. Seminars in Hematology, 47, 170-179.

https://doi.org/10.1053/j.seminhematol.2010.01.010

[13] Larrar, S., Guitton, C., Willem, M., et al. (2006) Severe Hematological Side Effects Following Rituximab Therapy in Children. Haematologica, 91, ECR36.

[14] Matthews, D.C. (2003) Rituximab in the Very Young: Benefits in AIHA, at What Risk? Blood, 101, 3761. https://doi.org/10.1182/blood-2003-03-0834

[15] Quartier, P., Tournilhac, O., Archimbaud, C., et al. (2003) Enteroviral Meningoencephalitis after Anti-CD20 (Rituximab) Treatment. Clinical Infectious Diseases, 36, e47-e49. https://doi.org/10.1086/345746

[16] Zhang, Z.N. and Shen, D. (2007) Diagnosis and Therapeutic Criteria of Hematologic Diseases. 3rd Edition, Science Press, Beijing, 3-70.

[17] Zent, C.S., Seereto, C.R., Laplant, B.R., et al. (2008) Direct and Complement Dependent Cytotoxicity in CLL Cells from Patients with High-Risk Early-Intermediate Stage Chronic Lymphocytic Leukemia (CLL) Treated with Alemtuzumab and Rituximab. Leukemia Research, 32, 1849-1856. https://doi.org/10.1016/j.leukres.2008.05.014

[18] Klepfish, A., Rachmilewitz, E.A., Kotsianidis, I., et al. (2008) Adding Fresh Frozen Plasma to Rituximab for the Treatment of Patients with Refractory Advanced CLL. QJM, 101, 737. https://doi.org/10.1093/qjmed/hcn085

[19] Michael, J.K., Susan, O.B., Maher, A., et al. (2005) Early Results of Chemoimmunotherapy Regimen of Fludarabine, Cyclophosphamide, and Rituximab as Initial Therapy for Chronic Lymphocytic Leukemia. Journal of Clinical Oncology, 18, 4079. https://doi.org/10.1200/JCO.2005.12.051

[20] William, W., Susan, O.B., Sijin, W., et al. (2005) Chemoimmunotherapy with Fludarabine, Cyclophosphamide, and Rituximab for Relapsed and Refractory Chronic 
Lymphocytic Leukemia. Journal of Clinical Oncology, 18, 1070. https://doi.org/10.1200/JCO.2005.12.516

[21] Brien, S.M., Kantarjian, H., Thomss, D.A., et al. (2001) Rituximab Dose-Escalation Trial in Chronic Lymphocytic Leukemia. Journal of Clinical Oncology, 19, 2165-2170. https://doi.org/10.1200/JCO.2001.19.8.2165

[22] Hainsworth, J.D., Litchy, S., Barrom, J.H., et al. (2003) Single-Agent Rituximab as First-Line and Maintenance Treatment for Patients with Chronic Lymphocytic Leukemia or Small Lymphocytic Lymphoma: A Phase II Trial of the Minnie Pearl Cancer Research Network. Journal of Clinical Oncology, 21, 1746-1751. https://doi.org/10.1200/JCO.2003.09.027

[23] Dorner, T. and Lipsky, P.E. (2007) B-Cell Targeting: A Novel Approach to Immune Intervention Today and Tomorrow. Expert Opinion on Biological Therapy, 7, 1287. https://doi.org/10.1517/14712598.7.9.1287

[24] Shanafelt, T.D., Madueme, H.I., Wolf, R.C., et al. (2003) Rituximab for Immune Cytopenia in Adults: Idiopathic Thrombocytopenic Purpura, Autoimmune Hemolytic Anemia, and Evans Syndrome. Mayo Clinic Proceedings, 78, 1340. https://doi.org/10.4065/78.11.1340

[25] Xu, J. and Qiu, H.Y. (2007) Rituximab Combined with Cyclosporine in the Treatment of Refractory Idiopathic Thrombocytopenic Purpura: A Case Report. Clinical Meta, 22, 1122.

[26] Zhou, Z.P. (2006) Clinical Progress of B Cell Elimination Therapy (Meruximab) in ITP. International Journal of Blood Transfusion and Hematology, 29, 495.

[27] Satoru, K. (2005) Rituximab Provided Long-Term Remission in a Patient with Refractory Relapsing Thrombotic Thrombocytopenic Purpura. International Journal of Hematology, 81, 433-436. https://doi.org/10.1532/IJH97.04187

[28] Sperr, W.R., Lechner, K. and Pabinger, L. (2007) Rituximab for the Treatment of Acquired Antibodies to Factor VIII. Haematologica, 92, 66.

https://doi.org/10.3324/haematol.10553

[29] Zeiser, R. and Blazar, B.R. (2017) Pathophysiology of Chronic Graft-versus-Host Disease and Therapeutic Targets. The New England Journal of Medicine, 377, 2565-2579. https://doi.org/10.1056/NEJMra1703472

[30] Li, X., et al. (2019) Developing Role of B Cells in the Pathogenesis and Treatment of Chronic GVHD. International Journal of Immunogenetics, 184, 323-336. https://doi.org/10.1111/bjh.15719

[31] Galimberti, S., Guerrini, F., Morabito, F., et al. (2003) Quantitative Molecular Evaluation in Autotransplant Programs for Follicular Lymphoma: Efficacy of in Vivo Purging by Rituximab. Bone Marrow Transplantation, 32, 57-63.

https://doi.org/10.1038/sj.bmt.1704102

[32] Fedoriw, Y., et al. (2012) Bone Marrow B Cell Precursor Number after Allogeneic Stem Cell Transplantation and GVHD Development. Annals of Surgical Treatment and Research, 18, 968-973. https://doi.org/10.1016/j.bbmt.2012.03.005

[33] Rehwald, U., Schulz, H., Reiser, M., et al. (2003) Treatment of Relapsed CD20+ Hodgkin Lymphoma with the Monoclonal Antibody Rituximab Is Effective and Well Tolerated: Results of a Phase 2 Trial of the German Hodgkin Lymphoma Study Group. Blood, 101, 420-424. https://doi.org/10.1182/blood.V101.2.420

[34] Hochster, H.S., Weller, E., Ryan, T., et al. (2004) Results of E1496: A Phase III Trial of CVP with or without Maintenance Rituximab in Advanced Indolent Lymphoma (NHL). Journal of Clinical Oncology, 22, 6502. 
https://doi.org/10.1200/jco.2004.22.90140.6502

[35] Dorner, T. and Lipsky, P.E. (2007) B-Cell Targeting: A Novel Approach to Immune Intervention Today and Tomorrow. Expert Opinion on Biological Therapy, 7, 1287. https://doi.org/10.1517/14712598.7.9.1287

[36] Li, J. and Wang, H.H. (2016) Simple Differential Diagnosis of Hematologic Diseases. People's Medical Publishing House, Beijing, 3-450. https://doi.org/10.18044/Medinform.201632.450

[37] Feucht, H.E., Schneeberger, H., Hillebrand, G., Burkhardt, K., Weiss, M., Reithmuller, G., Land, W. and Albert, E. (1993) Capillary Deposition of C4d Complement Fragment and Early Renal Graft Loss. Kidney International, 43, 1333-1338. https://doi.org/10.1038/ki.1993.187

[38] Vieira, C.A., Agarwal, A., Book, B.K., et al. (2004) Rituximab for Reduction of Anti-HLA Antibodies in Patients Awaiting Renal Transplantation: 1. Safety, Pharmacodynamics, and Pharmacokinetics. Transplantation, 77, 542-548. https://doi.org/10.1097/01.TP.0000112934.12622.2B

[39] Clifford, D.B., Ances, B., Costello, C., et al. (2011) Rituximab Associated Progressive Multifocal Leukoencephalopathy in Rheumatoid Arthritis. Archives of Neurology, 68, 1156-1162. https://doi.org/10.1001/archneurol.2011.103

[40] Genberg, H., Hansson, A.A., Wernerson, L., et al. (2007) Pharmacodynamics of Rituximab in Kidney Transplantation. Transplantation, 84, 33-36. https://doi.org/10.1097/01.tp.0000296122.19026.0f

[41] Celik, A., Saglam, F., Cavdar, C., et al. (2008) Successful Therapy with Rituximab of Refractory Acute Humoral Renal Transplant Rejection: A Case Report. Transplantation Proceedings, 40, 302-304. https://doi.org/10.1016/j.transproceed.2007.11.011

[42] Tanriover, B., Wright, S.E., Foster, S.V., et al. (2008) High-Dose Intravenous Immunoglobulin and Rituximab Treatment for Antibody-Mediated Rejection after Kidney Transplantation: A Cost Analysis. Transplantation Proceedings, 40, 3393-3396. https://doi.org/10.1016/j.transproceed.2008.08.131

[43] Faguer, S., Kamar, N., Guilbeaud-Frugier, C., et al. (2007) Rituximab Therapy for Acute Humoral Rejection after Kidney Transplantation. Transplantation, 83, 1277-1280. https://doi.org/10.1097/01.tp.0000261113.30757.d1

[44] Wade, E., Goral, S., Kearns, J., et al. (2006) Experience with Antibody Mediated Rejection in Kidney Allograft Recipients. Clinical Transplants, 10, 439-446. 